\title{
Cosmic-Ray Time Capsules
}

\author{
A proposed technique to study our Galaxy's cosmic-ray history involves \\ observing the damage created by neutrinos within deeply buried rocks.
}

By Philip Ball

$\prod$ he surface of Earth is constantly doused in cosmic rays, high-energy particles (mostly protons and light nuclei) from various astrophysical sources. Measuring how this flux has changed over billions of years could reveal clues about the sources and their evolution. Now, an international team of scientists has proposed a method for reading the signatures of cosmic rays in very ancient rocks from the deep Earth, extracted from geological boreholes [1]. The researchers show that damage to the crystal structures caused by particles produced by cosmic-ray collisions in the atmosphere could remain detectable in rocks as old as a billion years. These records might preserve signatures of ancient events such as supernovae in our cosmic neighborhood.

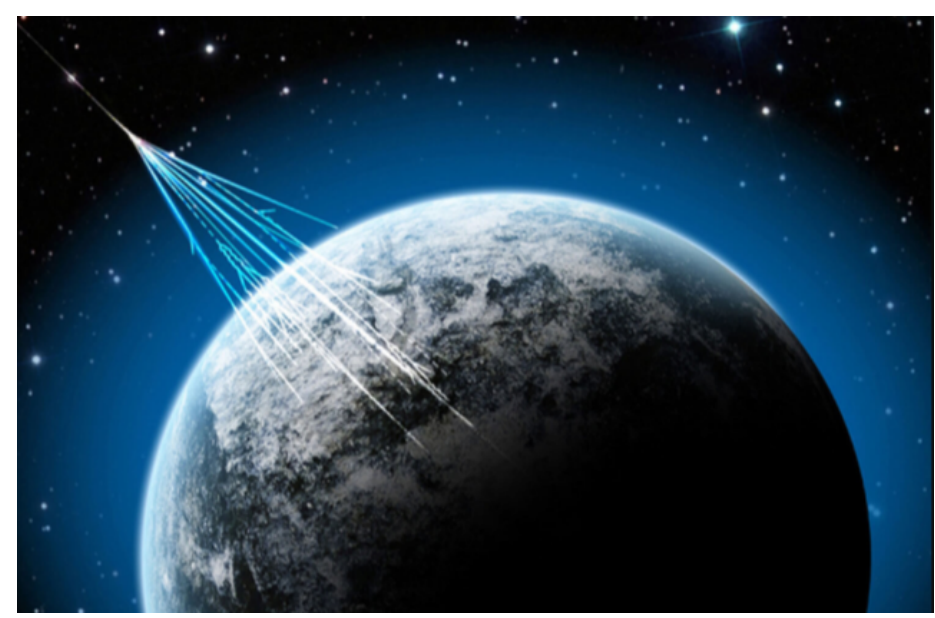

Relentless barrage. Cosmic rays collide with molecules in Earth's atmosphere, creating showers of particles that include neutrinos. The neutrinos can penetrate deep within Earth's surface, where they may leave a cosmic-ray record in buried rocks.

Credit: NSF/J.Yang
Scientists can reconstruct a historical record of cosmic-ray flux by measuring the concentration of the isotope beryllium-10 in rocks. The beryllium-10 is a product of cosmic-ray interactions in the atmosphere, but the timescale of its radioactive decay means that such records only go back a few million years. Neutrinos could offer a potentially longer record. Produced in the atmosphere by cosmic-ray collisions, these weakly interacting particles can pass deep into Earth before colliding with an atom in buried rock. Such a collision can potentially shatter the atom, causing knock-on damage in the form of tracks along which many atoms in the crystal are displaced in so-called nuclear recoils.

The idea of using nuclear-recoil tracks in ancient rocks as frozen signatures of exotic particles has a long history [2-6]. In particular, neutrino-initiated tracks have been proposed previously as records of past supernovae [7]. But up until now, no one had considered neutrino-initiated tracks as cosmic-ray markers. To understand whether this long-term record could be read in ancient, deep rocks, Josh Spitz of the University of Michigan, Ann Arbor, and colleagues have estimated how legible the tracks might be. Based on the numbers of atmospheric neutrinos generated by today's cosmic-ray flux, they say that 100 grams' worth of billion-year-old halite (sodium chloride, chosen as a simple example of a target rock) should contain about 60,000 neutrino-initiated tracks.

The researchers have used computer simulations and calculations to model the imprint that neutrinos would leave. The predicted tracks have a wide range of lengths, but those measuring 2-20 or 50-1000 micrometers should be distinguishable from background tracks produced by, for example, the decay of radioactive elements in Earth or by neutrinos coming from the Sun or nearby supernovae. To eliminate backgrounds from less-penetrating particles, such as 


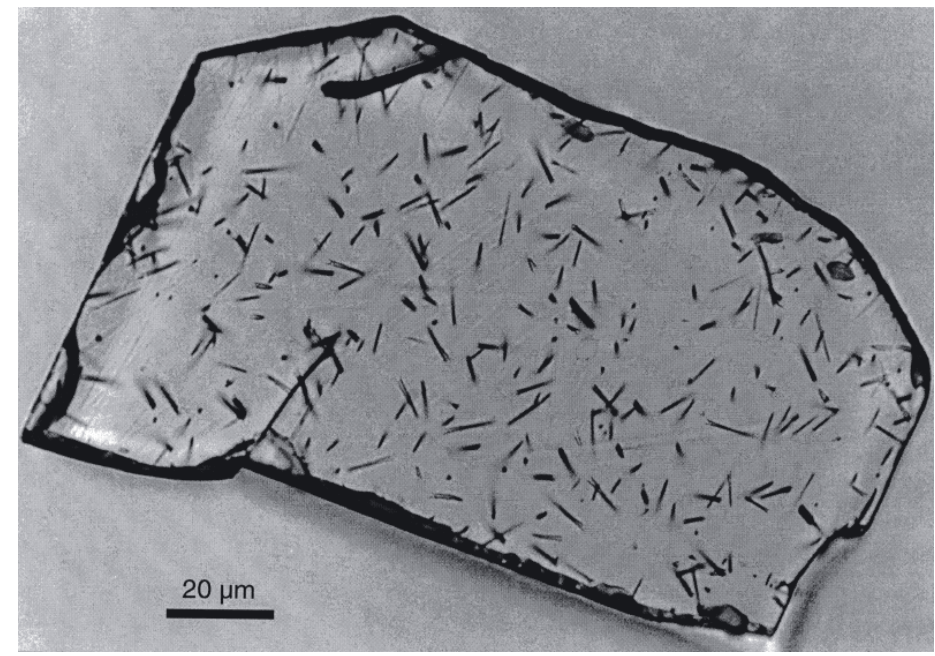

Cosmic tracks. This rock sample exhibits a large number of nuclear-recoil tracks coming from nuclear fission products. Researchers have proposed that rock samples from deep boreholes may contain similar tracks arising from neutrinos that originate in cosmic-ray interactions.

Credit: A. J. W. Gleadow et al., Rev. Mineral. Geochem. 48, 579

(2002)

cosmic-ray muons, the researchers recommend using rocks from depths below $5 \mathrm{~km}$. "Almost any mineral that is nonconducting can potentially form and retain tracks over geological timescales," says team member Patrick Stengel of Stockholm University.

The researchers show that, by measuring samples with a range of different ages over the past billion years or so, it should be possible to distinguish among, say, a constant cosmic-ray flux, one that steadily increases, and one in which there is a sudden but transient increase due to some cosmic event.

Determining the evolution in cosmic-ray flux could reveal possible trends associated with our Solar System's 250-million-year-long orbit around the Milky Way, says Michigan team member Johnathon Jordan. "Measurements going back a billion years would provide information about the past few orbits, which could tell us about the propagation and production of cosmic rays in our Galaxy."

There remains a lot of work to be done before the idea could be implemented, say Spitz and his colleagues. Laboratory measurements will be needed to verify models of track formation in controlled environments, and there are logistical challenges to collecting and analyzing deep rock samples from boreholes before their records become contaminated by surface exposure.

"The geophysical method proposed here would offer a novel way to probe past cosmic-ray activity," says astrophysicist Brian Fields of the University of Illinois at Urbana-Champaign. That activity could reveal our Galaxy's star formation history, which could be compared to other galaxies, he says.

Studies of meteorites have not shown clear evidence for any change in the cosmic-ray flux on timescales from millions to billions of years, says astrochemist Rainer Wieler of the Swiss Federal Institute of Technology (ETH) in Zurich. But such methods aren't sensitive to short-term variations. The new approach could avoid that limitation if enough precisely dated deep rocks can be found, says Wieler-but whether that can be done remains to be seen.

Philip Ball is a freelance science writer in London. His latest book is How To Grow a Human (University of Chicago Press, 2019).

\section{REFERENCES}

1. J. Jordan et al., "Measuring changes in the atmospheric neutrino rate over gigayear timescales," Phys. Rev. Lett. 125, 231802 (2020).

2. P. B. Price et al., "Search for grand-unified-theory magnetic monopoles at a flux level below the Parker limit," Phys. Rev. Lett. 52, 1265 (1984).

3. D. Snowden-Ifft et al., "Limits on dark matter using ancient mica," Phys. Rev. Lett. 74, 4133 (1995).

4. A. Drukier et al., "Paleo-detectors: Searching for dark matter with ancient minerals," Phys. Rev. D 99, 043014 (2019).

5. T. Edwards et al., "Digging for dark matter: Spectral analysis and discovery potential of paleo-detectors," Phys. Rev. D 99, 043541 (2019).

6. P. B. Price and M. H. Salamon, "Search for supermassive magnetic monopoles using mica crystals," Phys. Rev. Lett. 56, 1226 (1986).

7. S. Baum et al., "Paleodetectors for Galactic supernova neutrinos," Phys. Rev. D 101, 103017 (2020). 